\title{
Intercarrier Interference of Multiple Access UFMC with Flexible Subcarrier Spacings
}

\author{
Ljiljana Marijanović $^{\dagger}$, Stefan Schwarz ${ }^{\dagger \ddagger}$ and Markus Rupp ${ }^{\dagger}$ \\ $\dagger$ Institute of Telecommunications, Technische Universität (TU) Wien \\ $¥$ Christian Doppler Laboratory for Dependable Wireless Connectivity for the Society in Motion \\ Email: \{ljiljana.marijanovic,sschwarz,mrupp\}@nt.tuwien.ac.at
}

\begin{abstract}
In this paper we consider multi-user multicarrier transmissions with flexible subcarrier spacings. In a scenario of multiple users access with different subcarrier spacings the orthogonality between subcarriers is disrupted. The paper presents the Intercarrier Interference (ICI) that occurs between two users with different subcarrier spacings in a Universal Filtered Multicarrier (UFMC) system. We investigate the interference induced by one user to another. We propose closed form functions for ICI for both users and validate them with simulation results. Also, we provide simulation results of the ICI power in terms of filter lengths and number of guard subcarriers and we calculate the corresponding achieved time-frequency efficiencies.
\end{abstract}

Index Terms-Intercarrier interference, UFMC, guard subcarriers, subcarrier spacing, filter length

\section{INTRODUCTION}

Several transmission waveform contenders are currently considered within research and standardization for $5 \mathrm{G}$ wireless communications. Much effort has been invested in terms of choice and standardization of a new waveform by the 3rd Generation Partnership Project (3GPP), [1], [2], [3]. All proposed techniques have to be able to support the diverse set of requirements of future applications, according to their features, [4]-[9]. Many industry representatives consider Universal Filtered Multicarrier (UFMC) as the best choice for a multi-service air interface below $6 \mathrm{GHz}$, [2], [10], [11]. This scheme belongs to Orthogonal Frequency Division Multiplexing (OFDM)-based techniques but unlike cyclic prefix (CP)-OFDM, UFMC supports subband-wise filtering at the transmitter side, allowing more suppressed side lobes as well as a better fragmentation of the spectrum, [2], [12], [13]. The reduced side lobe level spectrum overcomes a problem of potential Intercarrier Interference (ICI) between users in case of asynchronous transmissions, [14].

UFMC offers flexibility in the sense that the assigned bandwidth can be divided into multiple subbands according to different user requirements or services [3], [6]. Services, such as, the exchange of safety relevant information in vehicular communications or tactile internet applications, require low latency transmission and, hence, a large subcarrier spacing. At the same time, cheap devices, such as, Internet of Things (IoT) sensors, may not be able to support very high sampling rates and thus require a small subcarrier spacing. Furthermore, a flexible subcarrier spacing enables the adaptation of the transmission waveform to channel properties, such as, the

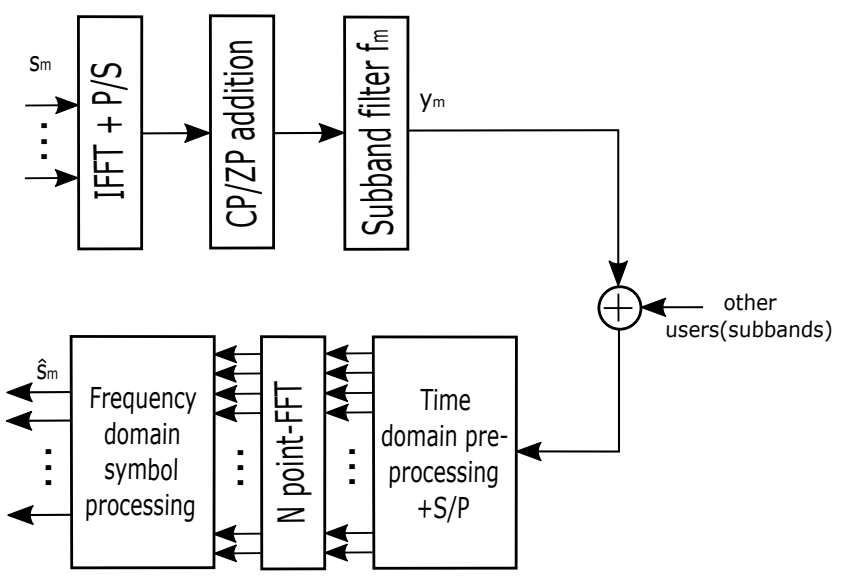

Figure 1: Basic UFMC transceiver

delay and Doppler spread of the channel to support reliable and efficient communication, [15], [16].

Hence, enabling a flexible subcarrier spacing is definitely of advantage in OFDM as well as in UFMC. However, if multiple subbands with different subcarrier spacing are concurrently transmitted, it causes ICI between the subbands since the orthogonality of the subcarriers is lost. In [17] the authors investigate this ICI, assuming two users are simultaneously served and taking twice the subcarrier spacing for one user in comparison to the other user. In order to decrease the amount of interference they provide a solution using smooth windows at the receiver with a raised-cosine, rather than a rectangular shape.

In this paper we also consider two users which occupy the entire bandwidth. One of the users has $15 \mathrm{kHz}$ subcarrier spacing which fits in the Long Term Evolution (LTE) structure (denoted as User 1), while the other user can use flexible subcarrier spacing (denoted as User 2). We derive closed-forms of ICI functions. Furthermore, we numerically evaluate ICI power in terms of different filter lengths, number of guard subcarriers and different subcarrier spacings.

\section{SYSTEM MODEL}

The basic transceiver structure is depicted in Figure 1, [18]. Vector $\mathbf{s}_{m} \in \mathbb{C}^{K_{m} \times 1}$ denotes the input symbols of subband $m$, where $K_{m}$ is the total number of subcarriers per subband. Since we assume that the bandwidth is occupied by two users, 
$m$ takes the maximum value of 2. Similiar to CP-OFDM, the frequency-domain input signal is then transformed to the time domain by an IFFT of length N. Instead of appending a cyclicprefix, however, we employ a zero prefix (ZP) following the proposal of [18]. The signal after the IFFT is denoted as:

$$
\mathbf{x}_{m}=\mathfrak{F}^{-1}\left\{\mathbf{s}_{m}\right\} \text {. }
$$

The main distinction of UFMC compared to CP-OFDM is subband filtering with a filter $\mathbf{f}_{m}=\left[f_{m}[0], f_{m}[1], \ldots, f_{m}[L-1]\right]$ where $L$ is the filter length. The filter has to be normalized to unit power. As we have already mentioned, the filtering yields better spectrum performance, enabling a good conservation of the spectrum. The signal after subband filtering is denoted by $\mathbf{y}_{m}$ and the total transmit signal is the sum of signals from individual subbands (users):

$$
\begin{gathered}
\mathbf{y}_{m}=\mathbf{x}_{m} * \mathbf{f}_{m}, \\
\mathbf{y}_{\text {total }}=\sum_{m} \mathbf{y}_{m} .
\end{gathered}
$$

In this paper, we intend to investigate the intrinsic ICI of the system due to the different subcarrier spacings. Hence, we consider an ideal channel without noise. In that case the received signal is:

$$
\mathbf{r}=\mathbf{y}_{\text {total }}
$$

This signal can be decomposed into two parts:

$$
\mathbf{r}=\left[\begin{array}{ll}
\mathbf{r}_{b o d y}^{T} & \mathbf{r}_{t a i l}^{T}
\end{array}\right]^{T}
$$

where $\mathbf{r} \in \mathbb{C}^{(N+L-1) \times 1}$ with $\mathbf{r}_{\text {body }}=\left[\begin{array}{ll}r_{0} & r_{1} \ldots r_{N-1}\end{array}\right]^{T}$ and $\mathbf{r}_{\text {tail }}=\left[\begin{array}{lll}r_{N} & r_{N+1} \ldots r_{N+L-2}\end{array}\right]^{T}$. In order to obtain a cyclic convolution (similar to CP-OFDM), we transform the received vector by copying the tail to the beginning of the signal:

$$
\hat{\mathbf{r}}=\mathbf{r}_{\text {body }}+\left[\begin{array}{ll}
\mathbf{r}_{\text {tail }}^{T} & \mathbf{0}_{[1 \times N-L+1]}
\end{array}\right]^{T} .
$$

This results in the same degree of complexity as CP-OFDM. It is possible to apply filtering or windowing before taking the FFT, which can reduce ICI in the scenarios, [17]. In the presence of a channel the equalization is indispensable and it has to be done immediately after taking the FFT. By processing all of those blocks, we successfully recover the transmit symbols, obtaining the recovered signal $\hat{\mathbf{s}}$. Since we do not apply filtering/windowing and consider an ideal channel without additive noise coefficients, we obtain:

$$
\hat{\mathbf{s}}=\mathfrak{F}\{\hat{\mathbf{r}}\},
$$

where simple extraction of subcarriers for corresponding subband gives us $\hat{\mathbf{s}}_{m}$.

The described signal processing steps can concisely be written as a matrix-valued input-output relationship:

$$
\hat{\mathbf{s}}_{m}=\mathbf{D}_{m} \boldsymbol{\Xi} \mathbf{F}_{m} \mathbf{D}_{m}^{H} \mathbf{s}_{m}
$$

with $\mathbf{F}_{m} \in \mathbb{C}^{(N+L) \times N}$ denoting a filtering matrix for subband $m$ corresponding to (2), $\boldsymbol{\Xi} \in \mathbb{C}^{N \times(N+L)}$ denoting a CP matrix at the receiver side, corresponding to (6) and $\mathbf{D}_{m} \in \mathbb{C}^{K_{m} \times N}$ denoting that part of a square DFT matrix $\mathbf{D} \in \mathbb{C}^{N \times N}$ that corresponds to the appropriate subband $m$.

\section{ICI INVESTIGATION}

\section{A. Motivation}

Let's consider a scenario with two users, where both use different subcarrier spacings; here we face ICI at the receiver side even with CP-OFDM. For all subsequent explanations we assume twice the subcarrier spacing for User 2 compared to User 1, unless stated otherwise. Since both users use different subcarrier spacing, they also apply a different FFT length for symbol detection: User 1 applies an $N_{1}$-FFT (denoted as Receiver 1) and User 2 applies an $N_{2}=N_{1} / 2$-FFT (denoted as Receiver 2). Since User 2 employs twice the subcarrier spacing of User 1, his useful symbol duration as well as ZP length are half as long as that of User 1. Hence, two short symbols of User 2 correspond to one symbol of User 1 :

$$
\begin{aligned}
\mathbf{x}_{1}^{(1)} & =\left[x_{1}^{(1)}(0), x_{1}^{(1)}(1), \ldots x_{1}^{(1)}\left(N_{1}-1\right)\right]^{T} \in \mathbb{C}^{N_{1} \times 1} \\
\mathbf{x}_{2}^{(1,2)} & =\left[\begin{array}{c}
\mathbf{x}_{2}^{(1)} \\
\mathbf{x}_{2}^{(2)}
\end{array}\right] \\
& =\left[\begin{array}{l}
{\left[x_{2}^{(1)}(0), x_{2}^{(1)}(1), \ldots x_{2}^{(1)}\left(N_{2}-1\right)\right]^{T}} \\
{\left[x_{2}^{(2)}(0), x_{2}^{(2)}(1), \ldots x_{2}^{(2)}\left(N_{2}-1\right)\right]^{T}}
\end{array}\right] . \in \mathbb{C}^{2 N_{2} \times 1}
\end{aligned}
$$

The signals received at both users are the sums of the transmit signals of the individual users:

$$
\mathbf{y}_{\text {total }}=\mathbf{x}_{\text {total }}=\mathbf{x}_{1}^{(1)}+\mathbf{x}_{2}^{(1,2)} .
$$

Considering an ideal channel and assuming a scenario without noise we can write the signal at Receiver 1 after FFT processing as:

$$
\hat{\mathbf{s}}_{1}^{(1)}=\mathfrak{F}\left(\mathbf{y}_{\text {total }}\right)=\mathbf{s}_{1}^{(1)}+\underbrace{\mathfrak{F}\left\{\mathbf{x}_{2}^{(1,2)}\right\}}_{\text {ICI }},
$$

where the ICI refers to the interference caused by User 2 .

$$
\begin{aligned}
\operatorname{ICI}(k) & =\sum_{n=0}^{N_{1}-1} \mathbf{x}_{2}^{(1,2)}(n) e^{\frac{-j 2 \pi k n}{N_{1}}} \\
& =\sum_{n=0}^{N_{2}-1} \mathbf{x}_{2}^{(1)}(n) e^{\frac{-j 2 \pi k n}{N_{1}}}+\sum_{n=N_{2}}^{N_{1}-1} \mathbf{x}_{2}^{(2)}(n) e^{\frac{-j 2 \pi k n}{N_{1}}} .
\end{aligned}
$$

Let us consider the second sum from (12):

$$
\begin{aligned}
\sum_{n=N_{2}}^{N_{1}-1} \mathbf{x}_{2}^{(2)}(n) e^{\frac{-j 2 \pi k n}{N_{1}}} & =\sum_{n=N_{2}}^{N_{1}-1} \sum_{k^{\prime}=0}^{N_{2}-1} \mathbf{s}_{2}^{(2)}\left(k^{\prime}\right) e^{\frac{-j 2 \pi k n}{2 N_{2}}} e^{\frac{j 2 \pi k^{\prime} n}{N_{2}}} \\
& =\sum_{k^{\prime}=0}^{N_{2}-1} \mathbf{s}_{2}^{(2)}\left(k^{\prime}\right) \sum_{n=N_{2}}^{N_{1}-1} e^{\frac{-j 2 \pi n}{N_{2}}\left(\frac{k}{2}-k^{\prime}\right)}
\end{aligned}
$$


where $k$ and $k^{\prime}$ denote different subcarrier spacing indices and $n$ is a time index. From (13) it is obvious that we cannot cancel out the second sum for $k$ odd. In that case orthogonality is lost, implying that User 2 causes ICI on each second subcarrier of User 1. For $k$ even, this sum vanishes due to the resource allocation orthogonality and thus ICI is not present. The same holds true for the first sum in (12). We can also prove the opposite case; in that case we can see that ICI is present even in each subcarrier of User 2. By increasing the subcarrier spacing at User 2 the amount of ICI will increase. We show this in the next section.

\section{B. ICI in UFMC}

Observing the UFMC system, we can control ICI by adjusting the length of the subband filter, which determines the decay of side lobes. Smaller filter lengths provide less suppression of side lobes in spectrum and correspondingly we have higher ICI. There are several criteria for the filter design in UFMC, [10], [19]. We consider a Hanning filter since it shows the best side-lobe attenuation compared to Hamming and Chebyshev filters. Inserting guard subcarriers between users is another helpful way to reduce ICI. Of course, both approaches cause a reduction of spectral efficiency. We investigate this in Section IV.

We derive the mathematical form of the ICI at Receiver 1 from the spectrum of User 2 that is shown in Figure 2. This is simply obtained as multiplication of the power spectrum density (PSD) of an OFDM transmit signal and the squaredabsolute value of the transfer function of the filter. This example is done for one resource block that comprises six subcarriers. In the general case the ICI has the following shape:

$$
I C I_{[d B]}(\omega)=10 \log _{10}\left[\sum_{k^{\prime}=0}^{K_{m}-1}\left|\operatorname{sinc}\left(\left(k^{\prime}+1\right) \frac{\pi}{2}+\frac{\omega}{2 q}\right)\right|^{2}|f(\omega)|^{2}\right],
$$

where

$$
f(\omega)=A\left(\omega, N_{2}, L_{2}\right) \operatorname{sinc}\left(\frac{\omega L_{2}}{2 q N_{2}}\right),
$$

with

$$
A\left(\omega, N_{2}, L_{2}\right)=\frac{c_{1}\left(N_{2}, L_{2}\right) \pi^{2}}{c_{2}\left(N_{2}, L_{2}\right) \pi+\omega^{2}},
$$

denoting the amplitude function of the filter depending on coefficient functions $c_{1}$ and $c_{2}$, determined by the FFT $N_{2}$ and filter length $L_{2}$, as well as angular frequency $\omega$. As can be seen, the ICI function is composed of two parts, one representing the pure OFDM part that depends on subcarrier index $k^{\prime}$, subcarrier spacing index $q$ and angular frequency $\omega$, and the other part describing the filter function, $f(\omega)$. The parameter $q$ represents the subcarrier spacing index, whose values $q=1,2, \ldots$ correspond to $15 \mathrm{kHz}, 30 \mathrm{kHz}, \ldots$ subcarrier spacing of User 2, respectively.

However, at Receiver 2 we apply an $N_{2}$-FFT only to half of the symbols transmitted by User 1 since the symbol duration of User 2 is half of that of User 1. This corresponds to a windowing of symbols from User 1 with a rectangular window in the time domain, and hence to a convolution with a sinc

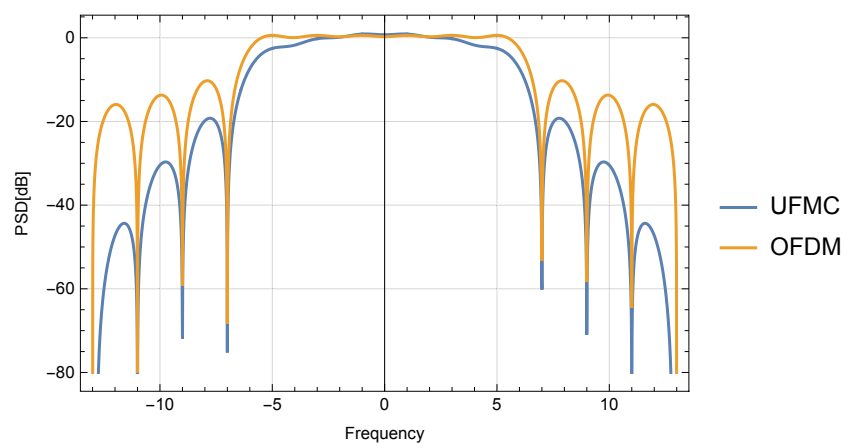

Figure 2: Spectrum of UFMC and OFDM signal of User 2.

function in the frequency domain, [17]. This convolution does not allow for closed-form analytic expressions. We hence utilize our vector-valued input-output relationship:

$$
\begin{aligned}
& \hat{\mathbf{s}}_{1}^{(1)}=\left[\mathbf{I}_{N_{2}}, \mathbf{0}_{N_{2}}\right] \overline{\mathbf{D}}_{2} \boldsymbol{\Xi} \mathbf{F}_{1} \mathbf{D}_{1}^{H} \hat{\mathbf{s}}_{1} \in \mathbb{C}^{N_{2} \times 1},
\end{aligned}
$$

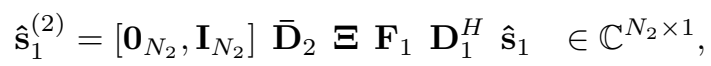

with identity matrix $\mathbf{I}$ of dimension $N_{2}$, a convolution matrix $\mathbf{F}_{1} \in \mathbb{C}^{\left(N_{1}+L_{1}\right) \times N_{1}}$ generated by a filter length of $L_{1}$ of User $1, \mathbf{D}_{1}$ as DFT matrix of dimension $N_{1}$ and CP matrix $\boldsymbol{\Xi} \in \mathbb{C}^{N_{1} \times\left(N_{1}+L_{1}\right)}$ :

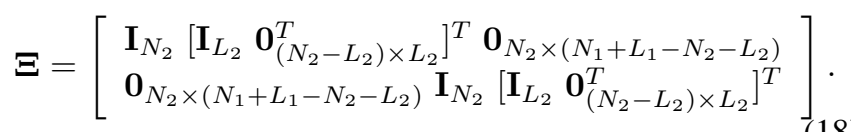

Matrix $\overline{\mathbf{D}}_{2}$ has the following shape:

$$
\overline{\mathbf{D}}_{2}=\left[\begin{array}{cc}
\mathbf{D}_{2} & \mathbf{0}_{N_{2}} \\
\mathbf{0}_{N_{2}} & \mathbf{D}_{2}
\end{array}\right]
$$

where $\mathbf{D}_{2}$ is a square DFT matrix of dimension $N_{2}$. In that case the ICI function is:

$$
I C I_{[d B]}(k)=10 \log _{10}\left[\frac{\left|\hat{\mathbf{s}}_{1}^{(1)}(k)\right|^{2}+\left|\hat{\mathbf{s}}_{1}^{(2)}(k)\right|^{2}}{2}\right] .
$$

\section{Simulation Results}

We consider a bandwidth of $10 \mathrm{MHz}$, occupied by two users. As we previously mentioned, User 1 has $15 \mathrm{kHz}$ subcarrier spacing and specifically we consider User 2 with $30 \mathrm{kHz}$ and $60 \mathrm{kHz}$ subcarrier spacing. In order to demonstrate that our analytical solution coincides with simulation results, we compare ICI powers with different filter lengths caused by User 2 that is obtained from analysis and simulation in Figure 3. Analytically, we observe the side lobe attenuation from the spectrum of User 2 that corresponds to the ICI power on the appropriate subcarrier index of User 1. It is important to mention that in this case we compare only one resource block of User 1 (12 subcarriers) and User 2 (6 subcarriers). Also, we calculate the sum of ICI power over all subcarriers within the resource block. Obviously the ICI gets higher as soon as the bandwidth is increased.

The ICI can be surely mitigated by applying longer filters as well as by introducing guard subcarriers. Longer and thus 


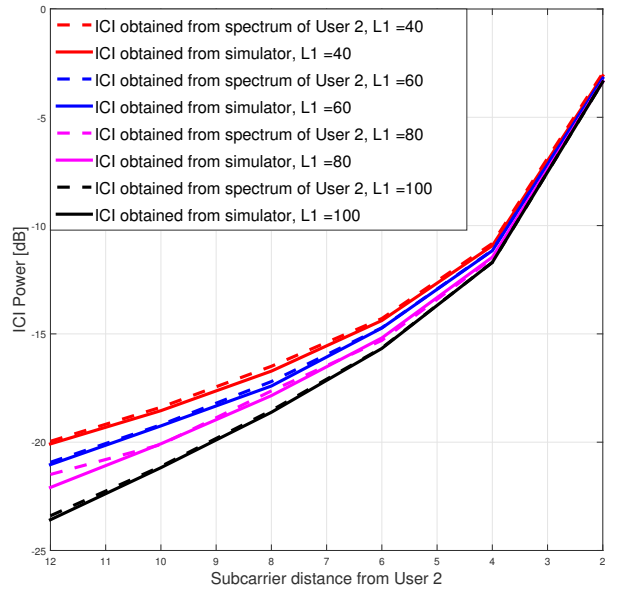

Figure 3: ICI at Receiver 1 obtained analytically and from simulation.

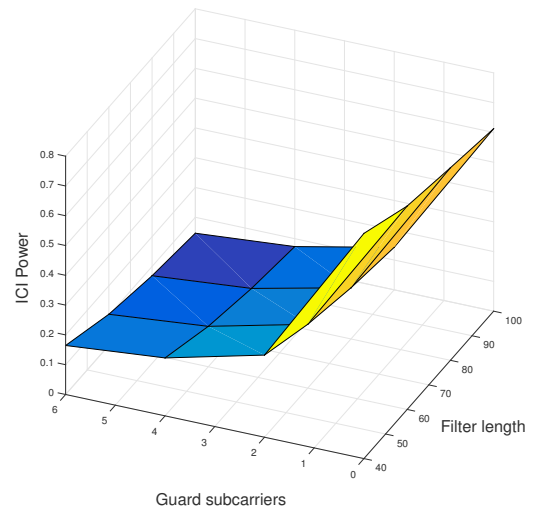

Figure 4: ICI at Receiver 1 in terms of guard subcarriers and filter length.

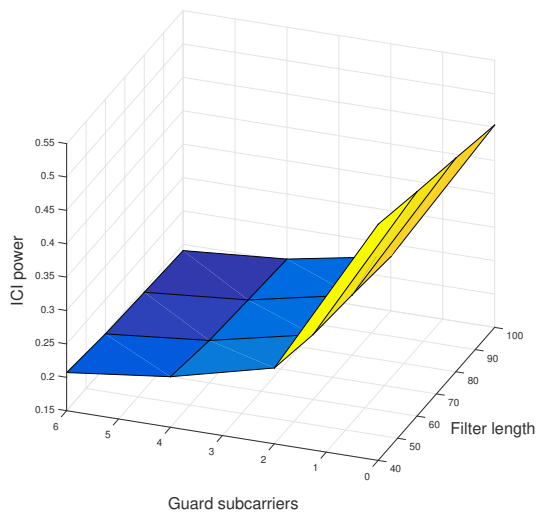

Figure 5: ICI at Receiver 2 in terms of guard subcarriers and filter length.

sharper filters decrease ICI. Also, with more guard subcarriers, ICI is more suppressed. This is confirmed in Figures 4 and 5 for both receivers. As we have previously mentioned User 1 experiences ICI on every second subcarrier, unlike User 2 who suffers from ICI on each subcarrier. We notice that the ICI of User 1 starts at a higher level for $L=0$ and zero guard subcarriers but decays with a faster rate compared to User 2 .

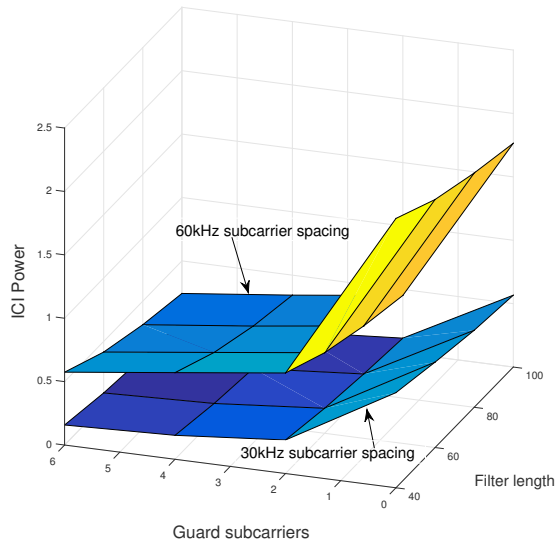

Figure 6: ICI at Receiver 1 for $30 \mathrm{kHz}$ and $60 \mathrm{kHz}$ subcarrier spacing.

In Figures 6 and 7 we compare the ICI of User 1 when User 2 employs a two and four times subcarrier spacing of User 1, i.e., $30 \mathrm{kHz}$ and $60 \mathrm{kHz}$, respectively. From these figures it is obvious that ICI increases as long as the subcarrier spacing increases. For instance, with $45 \mathrm{kHz}$ subcarrier spacing each third subcarrier of User 1 does not suffer from ICI, with $60 \mathrm{kHz}$ subcarrier spacing each fourth subcarrier. Also, we want to emphasize that $L_{60 \mathrm{kHz}}<L_{30 \mathrm{kHz}}$ and it has additional impact on ICI performance.

There is also a tradeoff between using the longer filters or assuming several guard subcarriers in terms of time-frequency efficiency; longer filter cause the loss of time efficiency due to the zero-padding while guard subcarriers are harmful for the frequency efficiency. The time-frequency efficiency is defined as:

$$
\mathrm{TF}=\frac{\mathrm{B}_{u}}{\mathrm{~B}} \frac{\mathrm{T}_{u}}{\mathrm{~T}} .
$$

where $\mathrm{B}_{u}$ is the useful bandwidth and $\mathrm{B}$ is the total bandwidth; $\mathrm{T}_{u}$ is the useful duration of the symbols and $\mathrm{T}$ is the entire symbols duration including the ZP length. In Figure 8 we compare the time-frequency efficiencies in terms of different filter lengths and different number of guard subcarriers. For the minimum filter length and zero guard subcarriers the smallest waste in both time and frequency domain is obtained. Unlike the ICI which quickly decreases with the number of guard subcarriers, the time-frequency efficiency decays slowly. Due to the filter lengths and at the same time the ZP lengths, the time efficiency has a higher impact in (21) compared to the frequency efficiency, Figure 8 . Of course, this depends on the total bandwidth $B$. Since we assume $10 \mathrm{MHz}$ bandwidth, a few subcarriers do not have a high impact on frequency efficiency. However, if the bandwidth is smaller, this impact is larger and thus the frequency efficiency is smaller.

\section{Conclusion}

In this paper, we provide analytical functions for the ICI between UFMC subbands using different subcarrier spacing. We derive two different ICI functions for the users with larger 


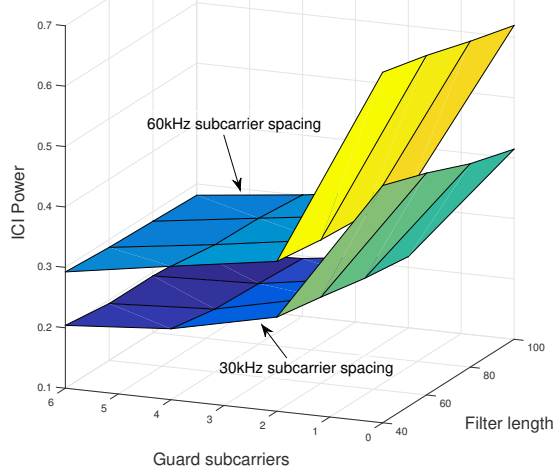

Figure 7: ICI at Receiver 2 for $30 \mathrm{kHz}$ and $60 \mathrm{kHz}$ subcarrier spacing.

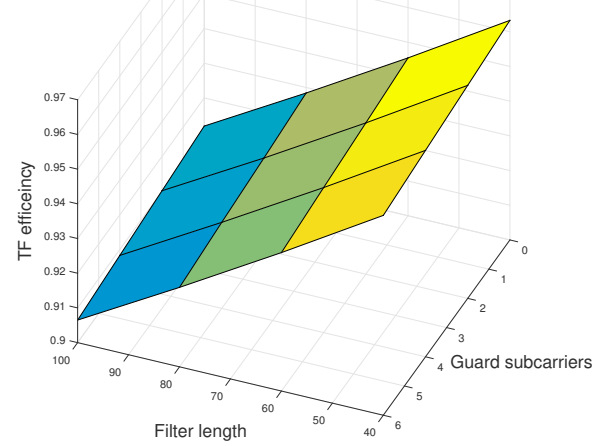

Figure 8: Time-frequency efficiency in terms of filter length and number of guard subcarriers.

and smaller subcarrier spacing, respectively. For the case we apply smaller subcarrier spacing the ICI is obtained from spectrum of User 2. In this case we observe that the amplitude function of the filter determines the side lobe attenuation. This function decreases quadratically when increasing the frequency, and due to that side lobes rapidly decline. For users with larger subcarrier spacing we utilize the vector-valued input-output structure of the system itself to achieve closed form of ICI.

UFMC offers flexibility in terms of subcarrier spacings but the problem of ICI when using multiple subbands with different subcarrier spacings simultaneously disrupts its performances. This is the most pronounced when applying a small filter length and omitting guard subcarriers at the same time. With the example of two users in this paper we want to show how different subcarrier spacings influence each other. Of course, a further division of bandwidth among more users brings new issues. Also, including the channel with different properties can additionally increase the ICI. Hence, the optimal solution should be found in order to suppress ICI while at the same time preserving time-frequency efficiency.
Acknowledgment: The financial support by the Austrian Federal Ministry of Science, Research and Economy and the National Foundation for Research, Technology and Development is gratefully acknowledged.

\section{REFERENCES}

[1] "R1-162199, Waveform candidates," 3GPP TSG-RAN WG1 \#84b, April 2016.

[2] "R1-165012, New radio waveforms for the multi-service air interface below $6 \mathrm{GHz}$, , 3GPP TSG-RAN WG1 \#85, May 2016.

[3] "R1-164655, Discussion on filtered waveform for NR," 3GPP TSG-RAN WG1 \#85, May 2016.

[4] N. Michailow, M. Matthé, I. S. Gaspar, A. N. Caldevilla, L. L. Mendes, A. Festag, and G. Fettweis, "Generalized frequency division multiplexing for 5th generation cellular networks," IEEE Transactions on Communications, vol. 62, no. 9, pp. 3045-3061, Sep 2014.

[5] R. Nissel and M. Rupp, "Enabling low-complexity MIMO in FBMCOQAM," in IEEE Globecom Workshops (GC Wkshps), Washington, USA, Dec 2016.

[6] X. Zhang, M. Jia, L. Chen, J. Ma, and J. Qiu, "Filtered-OFDM-enabler for flexible waveform in the 5th generation cellular networks," in 2015 IEEE Global Communications Conference (GLOBECOM), San Diego, CA, USA, Dec 2015, pp. 1-6.

[7] G. Berardinelli, K. Pajukoski, E. Lahetkangas, R. Wichman, O. Tirkkonen, and P. Mogensen, "On the potential of OFDM enhancements as 5G waveforms," in Vehicular Technology Conference (VTC Spring), 2014 IEEE 79th, Seoul, Korea, May 2014, pp. 1-5.

[8] T. Wild, F. Schaich, and Y. Chen, "5G air interface design based on universal filtered (UF-) OFDM," in International Conference on Digital Signal Processing (DSP), 2014 IEEE 19th, Hong Kong, China, Aug 2014, pp. 699-704.

[9] G. Wunder, P. Jung, M. Kasparick, T. Wild, F. Schaich, Y. Chen, S. Ten Brink, I. Gaspar, N. Michailow, A. Festag et al., "5GNOW: nonorthogonal, asynchronous waveforms for future mobile applications," IEEE Communications Magazine, vol. 52, no. 2, pp. 97-105, Feb 2014.

[10] "R1-164033, f-OFDM scheme and filter design," 3GPP TSG-RAN WG1 \#85, May 2016.

[11] "R1-165011, OFDM based Waveform for 5G new radio interface," 3GPP TSG-RAN WG1 \#85, May 2016.

[12] F. Schaich, T. Wild, and Y. Chen, "Waveform contenders for 5Gsuitability for short packet and low latency transmissions," in 2014 IEEE 79th Vehicular Technology Conference (VTC Spring), Seoul, Korea, May 2014, pp. 1-5.

[13] L. Zhang, A. Ijaz, P. Xiao, M. A. Imran, and R. Tafazolli, "MU-UFMC system performance analysis and optimal filter length and zero padding length design," arXiv preprint arXiv:1603.09169, March 2016.

[14] V. Vakilian, T. Wild, F. Schaich, S. ten Brink, and J.-F. Frigon, "Universal-filtered multi-carrier technique for wireless systems beyond LTE," in 2013 IEEE Globecom Workshops (GC Wkshps), Atlanta, GA, USA, Dec 2013, pp. 223-228.

[15] F. Schaich, T. Wild, and R. Ahmed, "Subcarrier spacing-how to make use of this degree of freedom," in Vehicular Technology Conference (VTC Spring), 2016 IEEE 83rd, Nanjing, China, May 2016, pp. 1-6.

[16] S. Schwarz and M. Rupp, "Society in motion: Challenges for LTE and beyond mobile communications," IEEE Communications Magazine, Feature Topic: LTE Evolution, vol. 54, no. 5, pp. 76-83, May 2016.

[17] F. Schaich and T. Wild, "Subcarrier spacing-a neglected degree of freedom?" in 2015 IEEE 16th International Workshop on Signal Processing Advances in Wireless Communications (SPAWC), Stockholm, July 2015, pp. $56-60$

[18] "R1-165014, Subband-wise filtered OFDM for new radio below $6 \mathrm{GHz}$," 3GPP TSG-RAN WG1 85, May 2016.

[19] S. Geng, X. Xiong, L. Cheng, X. Zhao, and B. Huang, "UFMC system performance analysis for discrete narrow-band private networks," in International Symposium on Microwave, Antenna, Propagation, and EMC Technologies (MAPE), 2015 IEEE 6th, Shanghai, China, Oct 2015, pp. 303-307. 